\title{
Interferometric reservoir monitoring with a single passive source
}

Carlos Almagro Vidal*, Joost van der Neut and Kees Wapenaar, Delft University of Technology

\section{SUMMARY}

Changes in the subsurface can be imaged by subtracting seismic reflection data at two different states, one serving as the initial survey or base, and the second as the monitor survey. Conventionally, the reflection data are acquired by placing active seismic sources at the acquisition surface. Alternatively, these data can be acquired from passive sources in the subsurface, using seismic interferometry. Unfortunately, the reflection responses as retrieved by seismic interferometry inherit an imprint of the passive source distribution. Therefore, monitoring with seismic interferometry requires high passive source repeatability, which is often not achievable in practice. We propose an alternative, by using active seismic data for the base survey and a single passive source for the monitor survey. By constraining the radiation pattern of the (active) base survey according to the characteristics of the (passive) monitor survey, we succeed to extract time-lapse response in the image domain. The proposed method is illustrated with numerically modeled data.

\section{INTRODUCTION}

With seismic interferometry (SI), a reflection response of the subsurface can be retrieved by cross-correlating the recordings of passive seismic sources. However, this method is based on several strong assumptions: noise sources should be uniformly distributed and mutually uncorrelated (Wapenaar and Fokkema, 2006). In practice, these conditions are generally not fulfilled (Draganov et al., 2009) and, as a consequence, the retrieved reflection response inherits an imprint of the passive source distribution. Removing this imprint from the recordings is a nontrivial task and requires solving an inverse problem, which can be highly ill-posed (Wapenaar and van der Neut, 2010; van Groenestijn and Verschuur, 2010). If passive illumination conditions are poor, for instance in cases where only a single passive source is available, this problem is unsolvable and alternative solutions are demanded.

The correlation gather of a single passive source contains correlated events induced by the presence of reflectors in the subsurface. These events are generally mispositioned in time, except for those traces that cover the so-called stationary-phase zone. In SI, destructive interference is achieved for all but those traces, whereas constructive interference takes place within the stationary-phase zone, eventually yielding the desired reflection response (Wapenaar and Fokkema, 2006). By using only the correlation gather of a single passive source, destructive interference can not be achieved. However, the gather can still provide useful information on the subsurface, assuming that it contains traces within the desired stationary-phase zone.

Almagro Vidal et al. (2012) presented a novel method to mi-

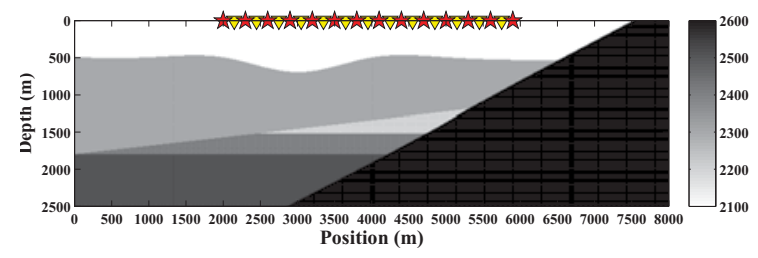

(a) State $A$

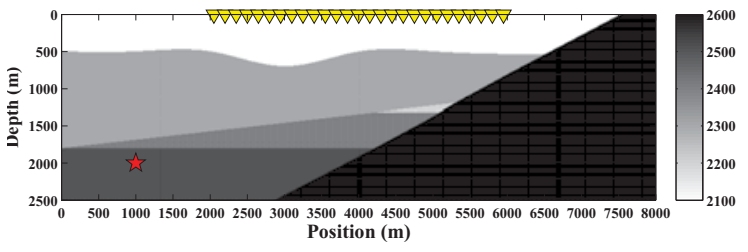

(b) State $B$

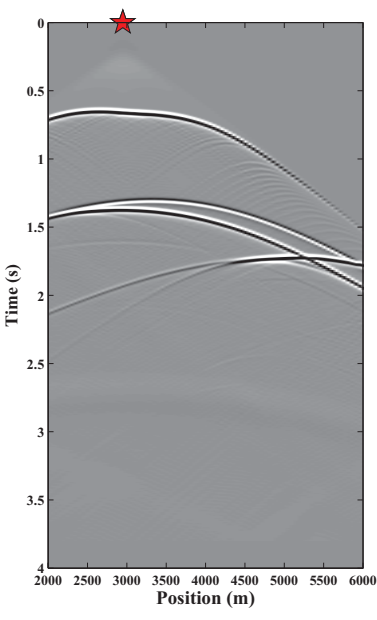

(c) $\bar{R}_{A}$

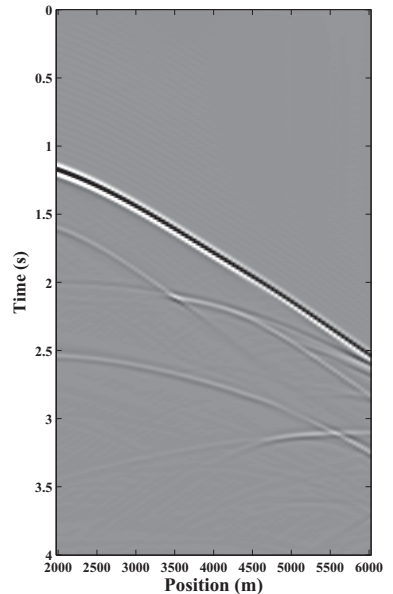

(d) $u_{B}$
Figure 1: (a) Velocity model $(\mathrm{m} / \mathrm{s})$ at state $A$ (base survey): controlled sources and receivers are located at the earth's surface between $2000 \mathrm{~m}$ and $6000 \mathrm{~m}$. (b) Model at state B (monitor survey): Notice the difference at the reservoir level at 1200 $m$ depth. The monitoring of this state is carried out using the same receiver array as in (a), but with a passive source in the subsurface (red star). (c) Reflection response in position $x^{\prime}=3000 \mathrm{~m}$ at state $A$ (as displayed in (a)), where the surfacerelated multiples have been removed $\left(\bar{R}_{A}\right)$. (d) Passive source recording at state $B\left(u_{B}\right)$, as illustrated in (b).

grate the correlation gather of a single passive source. The difference of this method to Schuster et al. (2004) is that contributions from outside the stationary-phase zone are avoided by constraining the directionality of the forward-propagated source field. Using this method for time-lapse applications re- 


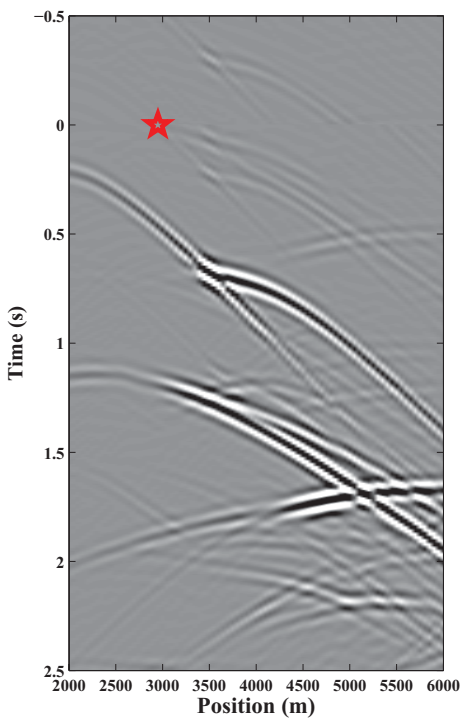

(a) $\mathbf{C}_{A, B}$

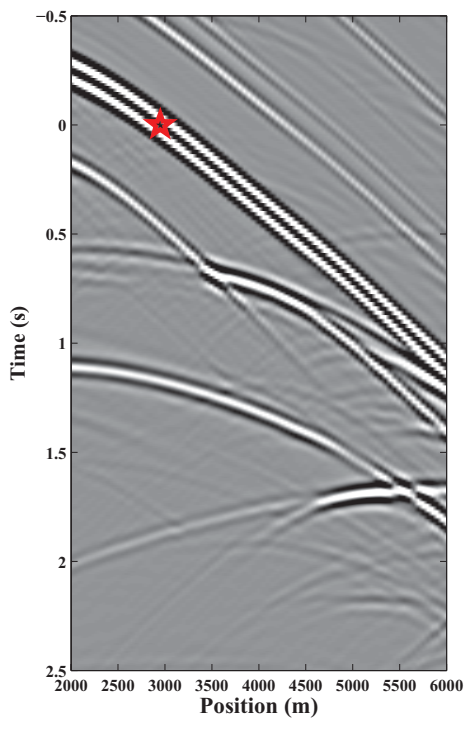

(b) $\Gamma_{B}$

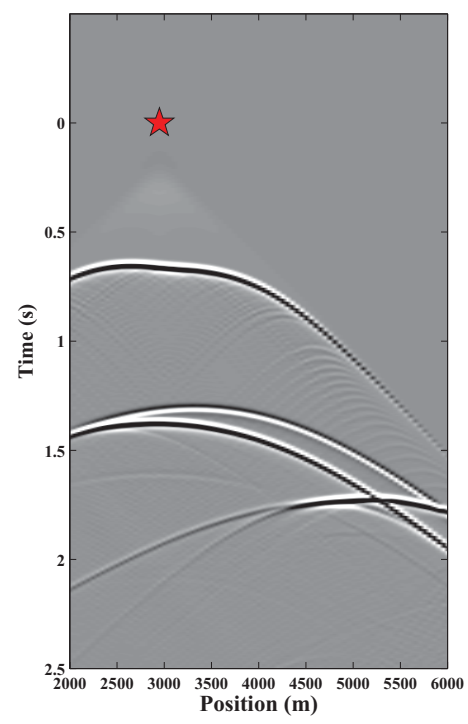

(c) $\overline{\mathbf{R}}_{A}$

Figure 2: (a) Correlation function $\left(\mathbf{C}_{A, B}\right)$ at virtual source $x^{\prime}=3000 \mathrm{~m}$, describing the state $A$ with the illumination induced from the passive source in state $B$. (b) Point-spread function $\Gamma_{\mathrm{B}}$ at $x^{\prime}=3000 \mathrm{~m}$ from the passive source recording $u_{\mathrm{B}}$ in state $B$ (see figures $1 \mathrm{~b}$ and $1 d$ ). (c) Reflection response $\bar{R}_{\mathrm{A}}$ at $x^{\prime}=3000 \mathrm{~m}$ in state A, without free-surface multiples.

quires passive source locations in the base and monitor survey to coincide. Since this is not feasible in practice, we propose an alternative method that requires controlled sources for the base survey and a single passive source for the monitor survey.

\section{THE CORRELATION FUNCTION}

Wapenaar et al. (2004) derived the following relation between the response of a general inhomogeneous medium without a free surface (indicated with a bar) and the same medium with a free surface (without a bar) in the frequency-space domain (where $\omega$ denotes the angular frequency):

$$
\bar{u}\left(\mathbf{x}, \mathbf{x}_{S}, \omega\right)-u\left(\mathbf{x}, \mathbf{x}_{S}, \omega\right)=\int_{\partial \mathbb{D}_{0}} \bar{R}\left(\mathbf{x}, \mathbf{x}^{\prime}, \omega\right) u\left(\mathbf{x}^{\prime}, \mathbf{x}_{S}, \omega\right) d \mathbf{x}^{\prime} .
$$

In this expression, $\partial \mathbb{D}_{0}$ is a depth level just below the free surface, $\bar{u}\left(\mathbf{x}, \mathbf{x}_{S}, \omega\right)$ is the transmission response at receiver $\mathbf{x}$ at the surface from a source at $\mathbf{x}_{S}$ in the subsurface, including all internal multiples but not the surface-related multiples. Further, $u\left(\mathbf{x}, \mathbf{x}_{S}, \omega\right)$ is the transmission response of the medium including all internal multiples and surface-related multiples. Finally, $\bar{R}\left(\mathbf{x}, \mathbf{x}^{\prime}, \omega\right)$ is the reflection response at receiver $\mathbf{x}$ from a source at $\mathbf{x}^{\prime}$ in a medium without free surface.

Equation 1 can be rewritten in the matrix-vector notation of Berkhout (1982) as

$$
\overline{\boldsymbol{u}}-\boldsymbol{u}=\overline{\mathbf{R}} \boldsymbol{u}
$$

where $\overline{\boldsymbol{u}}$ is a column vector containing $\bar{u}\left(\mathbf{x}, \mathbf{x}_{S}, \omega\right)$ for a single passive source location $\mathbf{x}_{S}$ and variable receiver locations $\mathbf{x}$ at $\partial \mathbb{D}_{0}$. Matrix $\overline{\mathbf{R}}$ contains data from $\bar{R}\left(\mathbf{x}, \mathbf{x}^{\prime}, \omega\right)$, where each column and row contain the data from an individual source and receiver, respectively.

Now, if we apply the adjoint (indicated by $\dagger$ ) of $\boldsymbol{u}$ to both sides of equation 2 , we obtain

$$
(\overline{\boldsymbol{u}}-\boldsymbol{u}) \boldsymbol{u}^{\dagger}=\overline{\mathbf{R}} \boldsymbol{u} \boldsymbol{u}^{\dagger}
$$

Assuming that the direct field $\overline{\boldsymbol{u}}$ can be extracted from the passive recording, the left-hand side of this expression can be computed. We refer to the result of this operation as the correlation function:

$$
\mathbf{C}=(\overline{\boldsymbol{u}}-\boldsymbol{u}) \boldsymbol{u}^{\dagger}
$$

Further we define the so-called point-spread function:

$$
\Gamma=\boldsymbol{u} \boldsymbol{u}^{\dagger}
$$

We can now substitute expressions 4 and 5 into equation 3 , leading to

$$
\mathbf{C}=\overline{\mathbf{R}} \Gamma
$$

According to this expression, the obtained correlation function of a single passive source can be interpreted as the reflection response without free surface $\overline{\mathbf{R}}$, convolved with the point-spread function $\Gamma$. 


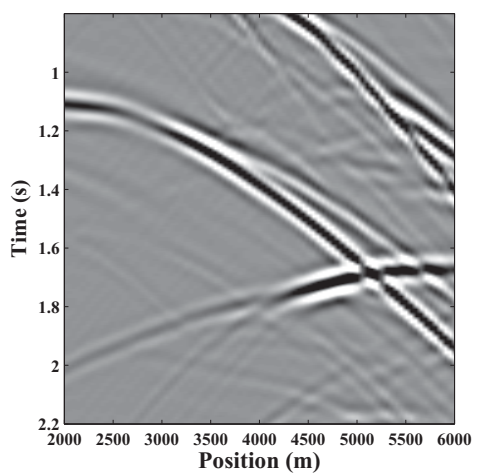

(a) $\mathbf{C}_{A, A}$

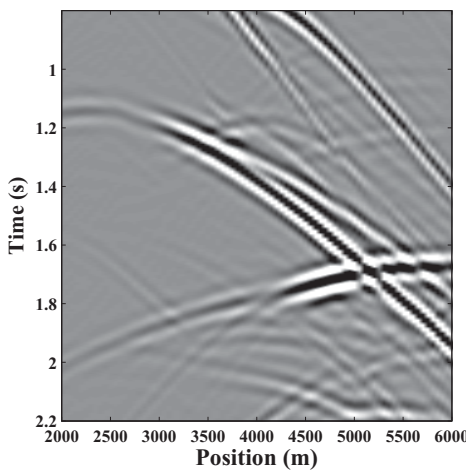

(b) $\mathbf{C}_{A, B}$

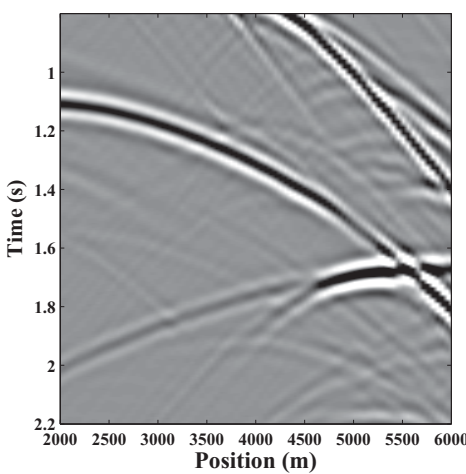

(c) $\mathbf{C}_{B, B}$

Figure 3: Close-up of the correlation functions at virtual source $x^{\prime}=3000 \mathrm{~m}$, at the reservoir time interval. (a) Correlation function of the passive source at State A, displayed for reference, $\left(\mathbf{C}_{A, A}\right)$. (b) Correlation function of the base with the induced illumination from the passive source at State $B\left(\mathbf{C}_{A, B}\right)$. (c) Correlation function of the monitor $\left(\mathbf{C}_{B, B}\right)$.

\section{PASSIVE MONITORING}

In this section, we aim to analyze changes in the subsurface between state $A$ and state $B$, represented in Figures $1 \mathrm{a}$ and $1 \mathrm{~b}$. In state A we have controlled sources available at the surface. The free-surface multiples and source wavelet should be eliminated from these data, either by surface-related multiple elimination (Verschuur et al., 1992) or by Estimation of Primaries by Sparse Inversion (van Groenestijn and Verschuur, 2009), providing us the reflection response $\overline{\mathbf{R}}_{A}$ in state $A$. The monitor survey in state $B$ is acquired from a single passive source recording $\boldsymbol{u}_{B}$. To obtain the correlation function of this source, we compute similar to equation 4 :

$$
C_{B, B}\left(\mathbf{x}, \mathbf{x}^{\prime}, \omega\right) \Rightarrow \mathbf{C}_{B, B}=\left(\overline{\boldsymbol{u}}_{B}-\boldsymbol{u}_{B}\right) \boldsymbol{u}_{B}^{\dagger} .
$$

In practice, $\overline{\boldsymbol{u}}_{B}$ is approximated by the time-windowed incident field of the passive source, where the contributions of the internal multiples are neglected. Remember from equation 6 that the correlation function can be interpreted as a multidimensional convolution of the desired reflection response of state $B$, $\overline{\mathbf{R}}_{B}$, and the point-spread function in state $B: \Gamma_{B}=\boldsymbol{u}_{B} \boldsymbol{u}_{B}^{\dagger}$.

To be able to compare the response of the base survey with the correlation function $\mathbf{C}_{B, B}$, we convolve $\overline{\mathbf{R}}_{A}$ with the obtained point-spread function of the monitor survey, yielding

$$
C_{A, B}\left(\mathbf{x}, \mathbf{x}^{\prime}, \omega\right) \Rightarrow \mathbf{C}_{A, B}=\overline{\mathbf{R}}_{A} \Gamma_{B} .
$$

First subscript in $\mathbf{C}_{A, B}$ represents the medium characteristics in state $A$, and second subscript represent the imprint of the point-spread function from state $B$. If we assume that the path between the source in the subsurface and the receivers at the surface do not cross the region where the changes take place, $\mathbf{C}_{A, B}$ and $\mathbf{C}_{B, B}$ inherit the imprint of the same point-spread function $\Gamma_{B}$, and therefore they can be directly compared to reveal the change of subsurface reflectivity between states $A$ and $B$, as we will demonstrate in the following example.

In Figure $2 \mathrm{a}$ we show the correlation function $\mathbf{C}_{A, B}$, obtained from the reflection response of the base survey $\overline{\mathbf{R}}_{A}$ (figure 2c) after convolution with the point-spread function $\Gamma_{B}$ (figure $2 b$ ). In Figure 3 we show a comparison of correlation functions at the reservoir level. The first panel, $\mathbf{C}_{A, A}$, is created for reference with a passive source in state $\mathrm{A}$ at the same location as the passive source that is actually located in state $B$. The second panel, $\mathbf{C}_{A, B}$, is generated by convolution of $\overline{\mathbf{R}}_{A}$ (obtained with controlled sources at the surface) with $\Gamma_{B}$. Note the similarities between figures $3 \mathrm{a}$ and $3 \mathrm{~b}$, indicating that the radiation patterns of the controlled sources in the base survey have been successfully modified to the radiation patterns that would be seen if these data were constructed from a passive source in the subsurface. The last panel, $\mathbf{C}_{B, B}$, is the correlation function as obtained from the passive source in state $B$. The difference of figure $3 \mathrm{c}$ and $3 \mathrm{~b}$ stems from the changes in the reservoir that we aim to retrieve.

\section{DIRECTIONALLY CONSTRAINED MIGRATION OF COR- RELATION FUNCTIONS}

Imaging of correlated data from passive sources has been presented in Almagro Vidal et al. (2012). In this method, the illumination characteristics serve as a directional constraint in the migration process, and only reflections that are in stationary phase are imaged. The illumination characteristics are studied using slowness analysis at the correlation of incident fields $\overline{\boldsymbol{u}}_{B} \overline{\boldsymbol{u}}_{B}{ }^{\dagger}$. This analysis defines the dominant ray-parameter which constrains the ray-path $\boldsymbol{p}_{B}^{\mathbf{x}}$ of the specular field from each virtual source location $\mathbf{x}^{\prime}$ at the surface. The forward-propagated source field is here constructed using the directional constraint given by $\boldsymbol{p}_{B}^{\mathbf{x}^{\prime}}$ :

$D_{B}^{\Downarrow}\left(\boldsymbol{p}_{B}^{\mathbf{x}^{\prime}}, \mathbf{x}_{0}, \mathbf{x}^{\prime}, t\right) \approx \frac{1}{\pi} \Re \int_{0}^{\infty} \hat{G}_{G B}\left(\boldsymbol{p}_{B}^{\mathbf{x}^{\prime}}, \mathbf{x}_{0}, \mathbf{x}^{\prime}, \omega\right) \hat{S}(\omega) e^{-i \omega t} d \omega$,

where $\hat{G}_{G B}\left(\boldsymbol{p}_{B}^{\mathbf{x}}, \mathbf{x}_{0}, \mathbf{x}^{\prime}, \omega\right)$ represents the Green's function representation using one single Gaussian beam with initial rayparameter $\boldsymbol{p}_{B}^{\mathbf{x}^{\prime}}$ at virtual source location $\mathbf{x}^{\prime}$ for any migration 


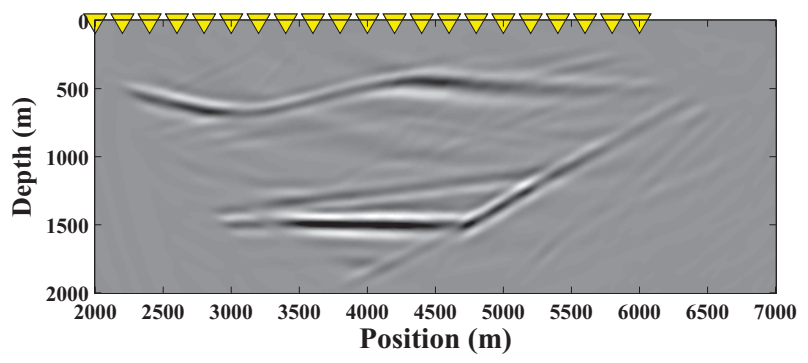

(a) $W_{A, A}$

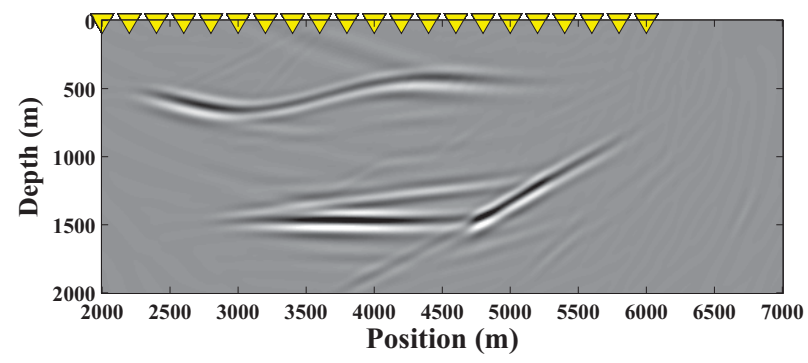

(b) $W_{A, B}$

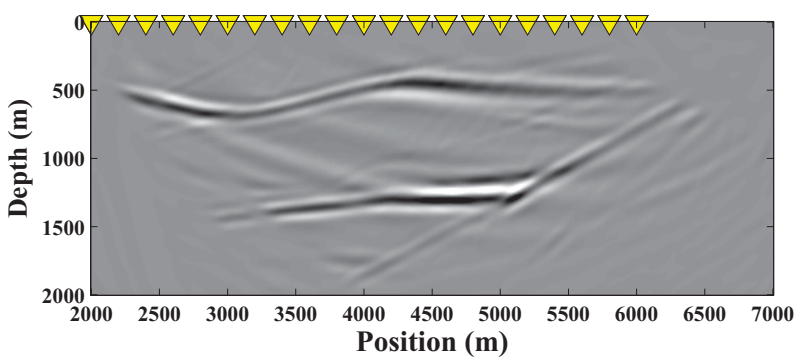

(c) $W_{B, B}$

Figure 4: Image results with different correlation functions. (a) Image result $W_{A, A}$ from the correlation function of the passive source at State $A\left(\mathbf{C}_{A, A}\right)$, serving as reference. (b) Image result $W_{A, B}$ of the correlation function obtained from the base survey with the induced illumination from the passive source $\left(\mathbf{C}_{A, B}\right)$. (c) Image result $W_{B, B}$ from the correlation function of the passive source $\left(\mathbf{C}_{B, B}\right)$. Notice the similarity between $(a)$ and $(b)$ : the reflectors have been illuminated only from those angles that could be provided by the passive source. This is achieved by constraining the radiation patterns of the controlled sources in the base survey and using these constrained radiation patterns in the migration scheme.

point $\mathbf{x}_{0} . \hat{S}(\omega)$ is the power spectrum of the correlation function $C_{A, B}$. We construct the backpropagated field with the Green's function representation using summation of Gaussian beams from every receiver location $\mathbf{x}$ in different directions, at every migration point $\mathbf{x}_{0}$, and evaluate at time $t_{0}$ :

$$
G_{G B}\left(\mathbf{x}_{0}, \mathbf{x}, t, t_{0}\right)=\frac{1}{\pi} \Re \int_{0}^{\infty} \hat{G}_{G B}\left(\mathbf{x}_{0}, \mathbf{x}, \omega\right) e^{-i \omega\left(t-t_{0}\right)} d \omega
$$

and now using the correlation functions obtained previously either for the base $C_{A, B}$ or the monitor $C_{B, B}$, the resulting backpropagated field is expressed as:

$$
\begin{aligned}
& U_{A, B}^{\Uparrow}\left(\mathbf{x}_{0}, \mathbf{x}^{\prime}, t_{0}\right) \\
& \quad \approx-2 \int_{t_{0}}^{T} \int_{\mathbf{x}} C_{A, B}\left(\mathbf{x}, \mathbf{x}^{\prime}, t\right) \frac{\partial}{\partial z} G_{G B}\left(\mathbf{x}_{0}, \mathbf{x}, t, t_{0}\right) d \mathbf{x} d t .
\end{aligned}
$$

Since all correlation functions are constructed using the same illumination characteristics, the imaging process uses the same source field for each image result. The imaging condition correlates $D_{B}^{\Downarrow}$ with $U_{A, B}^{\Uparrow}$ :

$$
W_{A, B}\left(\boldsymbol{p}_{B}^{\mathbf{x}}, \mathbf{x}_{0}, \mathbf{x}^{\prime}\right)=\int_{t_{0}}^{T} D_{B}^{\Downarrow}\left(\boldsymbol{p}_{B}^{\mathbf{x}^{\prime}}, \mathbf{x}_{0}, \mathbf{x}^{\prime}, t\right) U_{A, B}^{\Uparrow}\left(\mathbf{x}_{0}, \mathbf{x}^{\prime}, t\right) d t .
$$

In Figure 4 we display the image results using the correlated gathers from Figure 3 . The first panel, $W_{A, A}$, displays the reser- voir as it would be seen if the passive source were present in state $A$. The second panel, $W_{A, B}$, shows the same, obtained by cross-correlating the base survey $\overline{\mathbf{R}}_{A}$ with $\Gamma_{B}$. The final panel, $W_{B, B}$, shows the reservoir as seen by the passive source in state $B$. Changes in the wavelet phase from $W_{A, B}$ are due to inaccurate wavelet deconvolution of the synthetic data. The velocity model employed in all migration results is from state $A$ (See figure 1a). Note that the changes in the reservoir between figures $1 \mathrm{a}$ and $1 \mathrm{~b}$ can also be observed between figures $4 \mathrm{~b}$ and $4 \mathrm{c}$. We have thus successfully exposed the time-lapse response in the image domain, using a single passive source only.

\section{CONCLUSIONS}

We have shown that time-lapse signals can be retrieved in the image domain, using controlled sources at the surface as a base survey and a correlation function of a single passive source in the subsurface as a monitor survey. To do so, we had to modify the radiation patterns of the controlled sources in the base survey according to the illumination characteristics that were provided by the passive source in the monitor survey. Moreover, we applied a directionality-constrained migration scheme, where the forward-propagated source fields have been assigned these radiation patterns. 
http://dx.doi.org/10.1190/segam2013-1249.1

\section{EDITED REFERENCES}

Note: This reference list is a copy-edited version of the reference list submitted by the author. Reference lists for the 2013 SEG Technical Program Expanded Abstracts have been copy edited so that references provided with the online metadata for each paper will achieve a high degree of linking to cited sources that appear on the Web.

\section{REFERENCES}

Almagro Vidal, C., J. van der Neut, A. Verdel, D. Draganov, and K. Wapenaar, 2012, Passive interferometric imaging for limited illumination using slowness diagnosis and directionally constrained Gaussian beam migration: 82nd Annual International Meeting, Las Vegas, SPMI E$\mathrm{P} 1.6</$ bok $>$

$<$ bok>Berkhout, A. J., 1982, Seismic migration. Imaging of acoustic energy by wave field extrapolation, Elsevier. $</$ bok $>$

<jrn>Draganov, D., X. Campman, J. Thorbecke, A. Verdel, and K. Wapenaar, 2009, Reflection images from ambient seismic noise: Geophysics, 74, no. 5, A63-A67, http://dx.doi.org/10.1190/1.3193529. $</$ jrn>

Schuster, G. T., J. Yu, J. Sheng, and J. Rickett, 2004, Interferometric/daylight seismic imaging: Geophysical Journal International, 157, no. 2, Issue 2, 838-852, http://dx.doi.org/10.1111/i.1365246X.2004.02251.x.

van Groenestijn, G. J. A., and D. J. Verschuur, 2010, Estimation of primaries by sparse inversion from passive seismic data: Geophysics, 75, no. 4, SA61-SA69, http://dx.doi.org/10.1190/1.3460431.

van Groenestijn, G. J. A., and D. J. Verschuur, 2009, Estimating primaries by sparse inversion and application to near-offset data reconstruction: Geophysics, 74, no. 3, A23-A28, http://dx.doi.org/10.1190/1.3111115.

Verschuur, D. J., A. J. Berkhout, and C. P. A. Wapenaar, 1992, Adaptive surface-related multiple elimination: Geophysics, 57, 1166-1177, http://dx.doi.org/10.1190/1.1443330.

Wapenaar, K., and J. van der Neut, 2010, A representation for Green's function retrieval by multidimensional deconvolution: The Journal of the Acoustical Society of America, 128, no. 6, EL366-EL371, http://dx.doi.org/10.1121/1.3509797.

Wapenaar, K., 2008, Seismic interferometry-by-deconvolution for controlled-source and passive data: 70th EAGE Conference and Exhibition.

Wapenaar, K., and J. Fokkema, 2006, Green's function representations for seismic interferometry: Geophysics, 71, no. 4, SI33-SI46, http://dx.doi.org/10.1190/1.2213955.

Wapenaar, K., J. Thorbecke, and D. Draganov, 2004, Relations between reflection and transmission responses of three-dimensional inhomogeneous media : Geophysical Journal International, 156, no. 2, 179-194, http://dx.doi.org/10.1111/j.1365-246X.2003.02152.x. 